\title{
DBSMA: A MAC Protocol for Multi-hop Ad-hoc Networks with Directional Antennas
}

\author{
Sunil Suresh Kulkarni \\ School of Electrical and Computer Engineering, \\ Purdue University, West Lafayette, IN 47907-2035, USA. \\ Email: sunilkul@ecn.purdue.edu, Telephone: 765-494-0626
}

\author{
Catherine Rosenberg \\ Department of Electrical and Computer Engineering, \\ University of Waterloo, ON N2L 3G1, Canada. \\ Email: cath@ece.uwaterloo.ca, Telephone: 519-888-4016
}

\begin{abstract}
Various MAC protocols have been suggested for exploiting the possible benefits provided by directional antennas, but most of the solutions suggested are either based on the concept of sending (MAC level) control packets (such as RTS and CTS) in all directions, or based on the use of some omnidirectional control packet transmissions. We believe that these approaches fail short of exploiting the capability of directional transmission/reception fully and are incapable of handling all the problems resulting from the use of directional antennas in multi-hop wireless networks. Hence we first propose a set of requirements that should be met by a good MAC protocol that uses directional antennas. Then we propose a Directional Busy Signal Multiple Access (DBSMA) protocol that meets these requirements. In DBSMA, all the transmissions, receptions, and idle listening are directional. The need to listen in many directions when in an idle state is achieved by constantly changing the listen direction to cover the whole space. We also propose a novel directional back-off mechanism in which every node maintains independent back-off windows for each direction and show how it yields better performance. We show that DBSMA is well suited for ad-hoc multi-hop networks with directional antennas.
\end{abstract}

\section{INTRODUCTION AND RELATED WORK}

There have been many attempts to increase the throughput capacity of Wireless Local Area Networks (WLAN) to match the throughput of wired Local Area Networks (LAN). For this purpose, various standards in the IEEE 802.11 series have been developed which offer increasingly more data rates. The demand for higher data rates in WLAN has triggered a great interest in the design of better physical layers, in the use of directional antennas, and in the development of the associated MAC protocols. The commonly used MAC protocol in WLAN is Carrier Sense Multiple Access with Collision Avoidance (CSMA-CA) [1]. For example, the Distributed Coordinated Function (DCF) mode, i.e., the random access mode, in the IEEE 802.11 standard, uses a form of CSMA-CA.

Various studies [1]-[3] have shown that the simple CSMACA protocol has many drawbacks especially in a multi-hop environment and have proposed alternative solutions. One of the most serious drawbacks of this protocol is the hidden terminal problem. The hidden terminal problem can be addressed in part by exchanging short control messages before the actual data communication to reserve the channel. For example, the IEEE 802.11 protocol uses short control messages such as Request To Send (RTS) and Clear To Send (CTS). The RTS and CTS packets prevent collision of the DATA packets by virtually sensing and reserving (through Network Allocation Vector (NAV)) the communication channel in the vicinity of the sender and the receiver. A busy tone multiple access (BTMA) protocol [2], [4] and various modifications to it [2], [5] have also been proposed to solve the hidden terminal problem. Various other multichannel MAC protocols [2] have also been suggested. In multichannel protocols more than one communication and/or control channels are used. In [2] a NACK based protocol with a separate control channel for the RTS-CTS mechanism is proposed. Most of these solutions assume the use of omni-directional antennas.

While the use of directional antennas is common in other wireless areas, their use in multi-hop network is still very rare. However, it is expected that using directional antennas in a multi-hop environment could lead to better performance, e.g., higher data rates, reduced interference, and possibly higher spatial reuse. However in order to take full advantage of these potential benefits, we need to design MAC protocols that are directional antenna-friendly. This is the purpose of this paper.

Several researchers [6]-[8] have tried to address various issues related to the use of directional antennas in multihop networks. In [9], the authors propose a MAC protocol for directional antennas in which the sender node transmits a directional RTS. The CTS that follows this RTS request is sent in omni-directional mode. In the same paper, the authors propose to use an omni-directional RTS along with the directional RTS to reduce the probability of control packet collisions. They also propose to use a Directional WTS (Wait To Send) to improve the performance of their protocol. A directional multi-hop RTS mechanism is suggested in [7].

In [10], the authors propose a MAC protocol in which the RTS is sent directionally but in every direction. (We call this type of operation sweeping.) Thus effectively the omnidirectional RTS is reproduced using a directional antenna. The CTS that follows is directional. While the nodes use both directional RTS and directional CTS in [7], the nodes in [7] and [10] listen in omni-directional pattern when they are idle.

Based on the NAV concept, Directional NAV (DNAV) has been proposed in various papers ( [7], [10], [11]). The DNAV enables nodes to know which directions are blocked for how much time by other ongoing communications. This enables a better spatial reuse of the wireless channel but has several drawbacks. In particular, it is complex to implement and it is 


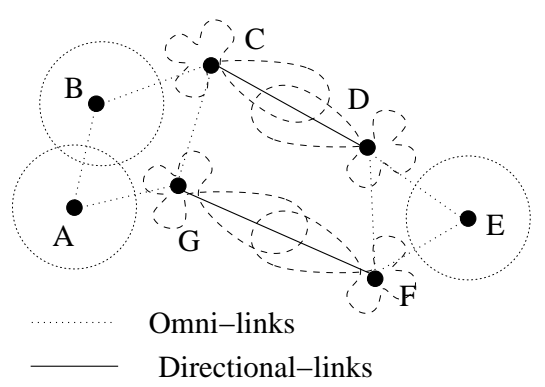

Fig. 1. Connectivity improvements using directional antennas

difficult to update the DNAV information for all directions as will be discussed later.

The paper is organized as follows. In Section II, we list the various features a good MAC protocol designed for directional antennas should have and point out the limitations of previous works with respect to these requirements. In Section III, we describe the proposed DBSMA protocol and explain its working in details. We present initial simulation results in Section IV.

\section{REQUIREMENTS FOR A MAC PROTOCOL USING DIRECTIONAL ANTENNAS}

Directional antennas potentially offer three kinds of benefits over omni-directional antennas:

1) Higher data rates and improved energy efficiency due to increased Signal to Interference and Noise Ratio (SINR)

2) Better connectivity due to improved communication range using directional pattern as shown in Figure 1 The directional patterns around the nodes are the radiation patterns, and not the communication range. For simplicity, in this paper we assume that two nodes are able to communicate whenever their radiation patterns intersect. The circular patterns around nodes A, B and $\mathrm{E}$ are the omni-directional patterns.

3) Improved spatial channel utilization due to directional communication. (Please refer to Figure 2): With omnidirectional antennas, an inactive pair of nodes in the vicinity of an active transmitter-receiver pair cannot start a new communication over the same channel. However, in some cases, by properly adjusting the (beam) directions of the directional antennas the inactive node pair might be able to start a new communication over the same channel. For example in Figure 2, while nodes $\mathrm{A}$ and $\mathrm{B}$ are communicating with each other nodes $\mathrm{C}$ and $\mathrm{D}$ can start another communication. Thus, the use of directional antennas provides a way to increase the spatial reuse of the channel.

These advantages are not independent of each other and to exploit them we need to consider them together rather than in isolation. Also these advantages are not without cost. Specifically, with directional antennas, overheads could be increased in terms of necessary information to be exchanged

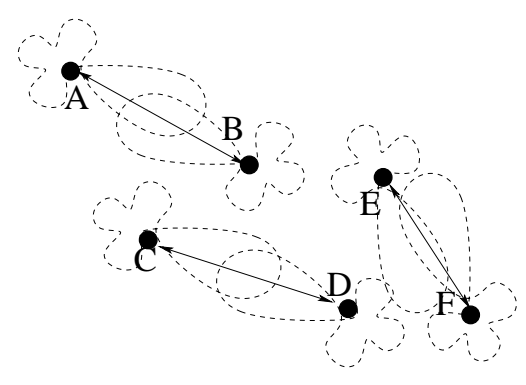

Fig. 2. Improvement in the spatial reuse using directional antennas

(such as node positions or antenna directions), required control packet transmissions (e.g. to adjust beam directions) and time and energy could be lost for steering the beams for example.

Before we discuss in detail the proposed DBSMA protocol, we list below the requirements for a good MAC protocol for directional antennas. We assume in the following that the antennas have a fixed transmission power that cannot be varied from transmission to transmission. This means that we are using directional antennas for improving the range of the communications and the spatial reuse as opposed to for decreasing the power consumption. Also we assume that the nodes can transmit, receive, and listen only in one (directional) pattern. For the directional antennas, we assume that the direction of the antenna can be maneuvered in discrete directions.

1) An effective protocol should use neither the omnidirectional transmission pattern nor the omni-directional idle reception pattern. If a protocol uses either of these then it amounts to losing some of the connectivity benefits offered by the directional antennas since for the same power, the range of any omni-directional action will be less than the corresponding unidirectional action. The only way to use a combination of omni and directional actions in an effective way is by adjusting the power level.

2) Sending directional control packets in each direction has energy and delay overheads. It also increases the probability of collision of control packets. Hence a good protocol must avoid directional transmissions in all directions even for control packets.

3) The protocol must not force all the nodes to have the same pattern for their antennas. This, in particular, implies that a node with an omni-directional antenna should be able to communicate with the same protocol to nodes with directional antennas.

4) The protocol must not assume an ideal directional antenna radiation pattern.

As noted in [10], the protocols suggested in [8], [9], [11] use at least one omni-directional control packet transmission. The protocols presented in [7], [10] use only directional transmissions and in this sense are closest to the proposed protocol in this paper, but they do not satisfy the other requirements as 
TABLE I

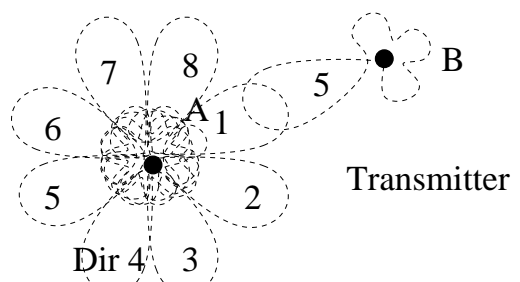

Idle receiver

Fig. 3. Behavior of an idle node in DBSMA protocol

follows. In both the papers, an idle node listens in an omnidirectional pattern (for example the RTS is received in an omni-directional mode) thus the first requirement is not satisfied. Hence this method cannot achieve maximum connectivity that is possible with the use of directional antennas. Also, the multi-hop RTS method suggested in [7] inherently assumes an ideal directional antenna/channel behavior for multi-hop RTS messages.

The DMAC protocol suggested in [10] requires multiple RTS transmissions for a single packet transmission which leads to an increased probability of control packet collision. As the directionality of the antenna increases, the energy and time overheads associated with this method also increase. In this protocol, the transmitter also listens for a CTS message in a omni-directional mode. This may increase the collision probability of RTS packets at the receiver. Another disadvantage of this protocol is that it requires every node to have an antenna with similar directional pattern, and a perfect directional orientation among all nodes. To effectively use this protocol, each node must know the state of each directional sector (through DNAV). However, the use of DNAV is itself a complex solution which ignores various factors such as the non-ideal propagation environment and the side lobe radiation patterns for directional antennas. Also it cannot ensure that the DNAV values are correctly updated at all nodes irrespective of their positions and listening directions because of directional communication patterns. For example, assume that the receiver node does not reply to the directional RTS. This information is not conveyed to the nodes in the other sectors in this protocol. Thus the DNAV for those nodes is not updated to take into account the missing CTS.

Table I summarizes the above discussion and enlists the characteristics of different protocols. DBSMA, the protocol proposed in this paper satisfies all the requirements of a good MAC protocol mentioned above. DBSMA is based on BTMA (busy tone multiple access) [4], that we have modified to make it work effectively for directional antennas.

The main contributions of this work are as follows. DBSMA solves the hidden terminal problem in the directional antenna setting. It is robust and it does not depend on the assumption of ideal directional pattern (for example, we do not assume that there are no side-lobe radiations) or ideal channel conditions. It can be followed by all nodes irrespective of their physical
SUMMARY OF VARIOUS MAC PROTOCOLS

\begin{tabular}{|c||c|c|c|c|c|c|}
\hline No. & Protocol & RTS S-Tx & CTS R-Tx & Data S-Tx & Data R-Rx & Idle Listen \\
\hline \hline 1 & CSMA-CA & NA & NA & Omni & Omni & Omni \\
\hline 2 & IEEE 802.11 & Omni & Omni & Omni & Omni & Omni \\
\hline 3 & DMAC [9] & Dir & Omni & Dir & Dir & Omni \\
\hline 4 & [8] & Omni & Omni & Dir & Dir & Omni \\
\hline 5 & DMAC & Dir & Dir & Dir & Dir & Omni \\
& \&MMAC [7] & multi-hop RTS & Omni \& Dir & Dir & Dir & Omni \\
\hline 6 & DMAC [10] & Dir & Dir & Dir & Dir & Omni \\
\hline 7 & DBSMA & Dir & Dir & Dir & Dir & Dir \\
\hline \multicolumn{7}{|c|}{} \\
\hline
\end{tabular}

antenna type, i.e., different nodes can have directional antennas with different beamwidths and even omni-directional antennas. Finally, we propose a directional back-off mechanism, in which the back-off window in one direction is independent of the back-off in any other direction. This mechanism yields better performance as will be shown later.

\section{Directional Busy Signal Multiple Access} (DBSMA)

In the previous section we argued that for achieving increased connectivity through directional antennas only directional operations should be performed. Hence in this paper, we assume that the nodes can transmit, receive, and listen only in a directional pattern. The direction of the antenna can be maneuvered in discrete directions. We also assume that, if at a node, a data transmission or data reception or idle listening is happening in a given direction, the node can also transmit, receive, or listen to a busy tone (a narrow band signal) in the same direction. To make the description easier, the fixed transmission power is assumed to be the same at each node even though this assumption can be easily relaxed.

Our DBSMA (Directional Busy Signal Multiple Access) protocol has the following features.

- A node, while in the idle state, sweeps all the directions continuously to listen, i.e., it rotates the hearing direction of its directional antenna continuously so as to listen in all directions. For example, if a node has a beamwidth of 45 degrees in a plane (as shown in Figure 3), then it would beamform its antenna in eight directions consecutively (e.g., node $\mathrm{A}$ in Figure 3 sweeps directions $1, \ldots, 8$ one after another). There are two reasons for designing DBSMA that way. i) We use the directional pattern to listen which gives us a larger listening range and hence we can exploit the connectivity benefit, ii) We have assumed that a node can only listen in a directional pattern.

- After receiving an RTS message, a receiver sends a unique directional CTS to the transmitter using the direction in which it received the RTS. It does not send any other control packets in any other directions.

- While in the reception mode, i.e., after receiving an RTS destined to it, the receiver continuously transmits a busy tone as in the BTMA protocol till the end of the DATA and ACK transmissions. The busy signal is sent with the same directional pattern as the receive directional pattern. The busy signal is a narrow bandwidth, out of band signal. 
- A node that wants to transmit, senses the channel in the direction of the receiver (i.e., the next-hop destination) and if the channel is perceived idle, it should attempt sending a unique directional RTS to the destination. However, before sending this RTS message, it sends an Invitation Signal (IS) in the same direction. The invitation signal is a narrow-band signal like a busy tone signal and is sufficiently long so that all the idle neighbors can listen to it (irrespective of their initial sweeping state), correctly determine the existence of the invitation signal, and lock on that direction for reception of the actual RTS message.

\section{A. Protocol Description}

The DBSMA protocol is partly based on the 802.11 MAC protocol, partly on the BTMA protocol, and partly new with the introduction of the invitation signal and the directional back-off. Suppose a node wants to send a packet to a neighboring node (e.g., node B wants to communicate with node A in Figure 3). Assume that the source knows the direction it should use to reach the destination. The source then sends an invitation signal and then an RTS with the correct directional antenna pattern to the destination. If a node is equipped with an omni-directional antenna instead of a directional antenna, it sends an invitation signal followed by an RTS message using the omni-directional pattern.

If the destination node has a directional antenna and if the directional antenna is not set to receive in the direction of the transmitter, the receiver node may not be capable of detecting the invitation signal even if it is idle.

To counter this problem, in the literature it has been suggested that the receiver node should always listen with an omni-directional antenna. But this poses some serious problems. Indeed, under our assumption of constant transmission power, a node listening with an omni-directional pattern would not be able to listen as far as it could with a directional pattern and hence may not hear an RTS coming from a node $\mathrm{B}$ which is out of range of the omni-directional pattern but in range of the directional pattern. Hence, this amounts to giving up some connectivity and reuse benefits provided by the directional antenna. Moreover, some other ongoing communications (which would not interfere if the receiver antenna is directional) may also adversely affect an omnireception. Clearly to exploit the directional antenna capability fully, we should not force the receiver to listen with an omnidirectional pattern. Hence we propose that, in an idle listening state, a node sweeps the whole space by rotating the direction of its antenna waiting to catch an invitation signal. This is shown in Figure 3. Node A in idle state is shown to change idle listening direction in sequence from direction 1 to direction 8 in anticipation of an invitation signal.

Hence, to initiate a data communication, before sending an RTS, a transmitter sends an Invitation Signal. The invitation signal can be implemented as a narrow-band signal on a separate channel or as a narrow-band signal somewhere in the data/control channel frequency band. The duration for the invitation signal is dimensioned such that any idle node within the range of a transmitter node can successfully receive this invitation signal irrespective of its initial sweeping state.

Let the total time required to beamform in one direction, to determine if there is any ongoing activity and to switch to a new direction be denoted as $r$. The time duration for the invitation signal should be greater than or equal to $2 \pi r / X=$ $r K$, where $X$ is the minimum beamwidth (in radians) among all nodes in the network and $\mathrm{K}$ is the maximum number of directional sectors for any node in the network. To minimize the energy consumption spent while transmitting the invitation signal and to obtain a higher channel utilization, it is necessary to design antennas with small $r$, i.e., the sweeping operation should be done as fast as possible while allowing any idle node in the vicinity to lock to an invitation signal. Thus, the time required to correctly determine the presence or the absence of the invitation signal and the switching time between two directions determine the maximum speed at which a node can sweep the whole space.

Whenever an idle node in a listening state detects an invitation signal, it locks to that direction and starts listening for a valid RTS. For example, in Figure 3, node A stops rotating direction once it detects the invitation signal on direction 1 . At the end of the transmission of the invitation signal, the transmitter sends a directional RTS. The nodes that receive this RTS and are not the intended receivers remain silent and can start sweeping again (see later for more details). On the other hand, once the receiver receives this RTS, it waits for a short period of time (called SIFS for Short Inter France Space in IEEE 802.11) and then sends a directional CTS to the transmitter. At the same time, it also starts sending a busy signal using the same directional pattern until the DATA and ACK packet transmissions between the two nodes finish. This enables the source/destination pair to correctly perform an RTS-CTS directional operation. The transmitter can begin the data transmission using its directional antenna after the RTS and the CTS have been successfully exchanged. In Figure 3, node A and node B are shown to be engaged in a communication using directions 5 and 1 respectively.

Before starting to send an invitation signal, a node has to listen to check if there is a busy tone or a busy data channel in the direction which would be used to communicate with the destination. Two things may happen: 1) If both channels are idle, the node computes a back-off time based on its current back-off window in that direction and sends an IS after that time if both channels are still idle. If not, the node will try again listening to both channels after some short time period and then start the process anew. 2) Otherwise, the transmitter concludes that it cannot communicate with the intended receiver as the receiver or some other receiver/transmitter pair in the vicinity is busy. In that case, it must remain silent till the busy signal channel and the communication channel become idle (i.e., the busy signal power and the communication channel signal power should drop below some threshold). Instead of waiting for the busy channel(s) to become idle, the sender may choose to communicate with another node in another direction (not necessarily for the same packet) and 
repeat the same procedure in the direction of the other node.

As a sender node listens to the busy signal before transmitting an RTS message, the receiver node also listens to the busy signal and communication channel in the corresponding direction before sending a CTS packet and a Busy Tone to avoid collision with some other ongoing communication.

As stated earlier, if the sender node does not sense any busy signal then it chooses to send an invitation signal followed by a RTS message. It may not get a CTS reply from the intended receiver because of three possibilities. i) The receiver is busy with the reception/transmission in another direction, ii) The receiver is blocked from transmission and unable to respond because a CTS response could disturb some other ongoing transmission, iii) There could have been an RTS collision (RTS packet in error). The transmitter then follows a directional back-off procedure and remains silent for some random time period. The directional back-off mechanism is explained in Section III-B.

Even if a node receives an RTS not destined to it, it can extract from it some useful information. Indeed, both the RTS and CTS carry a NAV field which can be used by the other nodes as a Directional NAV field. This information can be used by a node for scheduling the rotation of its beams (some sectors can be skipped for some time corresponding to the DNAV when sweeping for listening). Note that not all neighboring nodes would receive the DNAV (as they might be communicating with other nodes in other directions) and hence the use of the DNAV is limited. The busy signal serves similar purpose and enables a correct operation without the necessity of passing of the DNAV values to all neighboring nodes. Note that in [10] the transmitter node sweeps the entire space using directional antennas to transmit RTS in all directions while in DBSMA the idle nodes in a listening state sweep the entire space to listen. If $r$, the time period required for switching the beam direction and locking to the invitation signal is small compared to the time to switch and send an RTS in all directions, DBSMA is more energy efficient than DMAC. In any case, DBSMA reduces the number of control packet collisions, and does not require exact DNAV information for its correct operation.

\section{B. Enhancements to the DBSMA protocol}

In this section, we describe how to enhance the efficiency of our DBSMA protocol. These enhancements cannot be used with other BTMA protocols designed for omni-directional antenna, and this distinguishes DBSMA further from the BTMA protocol. These enhancements also cannot be used with most of the other directional MAC protocols such as [9], [10]. These enhancements have a major impact on the performance of a multi-hop ad-hoc networks with directional antennas as will be discussed later.

1) DBack-off: (Independent) Directional Back-off: The MAC protocols such as IEEE 802.11 provide a back-off mechanism so that the access to the channel by each node can be randomized and adapted to the current load on the wireless channel. A collision among control packets (usually
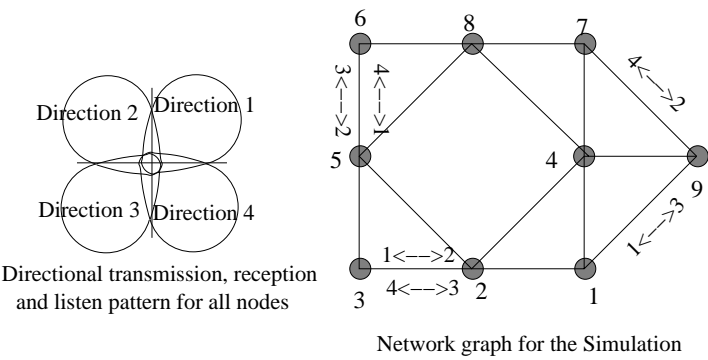

Fig. 4. Network graph for the simulations: Node 6 can communicate with node 5 in two ways - using direction pairs $(4,1)$ and $(3,2)$. For example, if node 6 uses direction 4 and node 5 uses direction 1 then both can communicate with each other. Similar direction pairs are assumed for other communication links.

RTS) increases the back-off window while a successful communication decreases it. This enables all the nodes to adapt to the current spatial network load.

Using directional antennas could give us the opportunity to adapt independently to the traffic conditions in different directions. For a node, one direction could be highly congested while the other directions might be less congested. Using the same back-off for all directions is an ill-fitted approach. A collision in one direction would then increase the backoff windows for the other directions which are potentially underutilized. Moreover, a successful transmission in an uncongested direction could decrease the back-off window in a congested direction as well. This has a two-fold effect on the channel utilization. First, it would decrease the channel utilization in the un-congested areas. And second, it would increase the probability of control packet collision in the congested areas thus increasing the back-off window. The coupling effects between these would make the network more unstable which would have an adverse effect on the quality of service.

Hence we propose to use an independent back-off for each direction. Essentially, a node with an antenna beamwidth of $\mathrm{X}$ (in radians) can be considered as a set of $2 \pi / X$, pseudo-nodes which are active in different directions. These pseudo-nodes run independent back-offs mechanisms. Alternatively, we may think that each direction behaves as if it is an independent link. Note that this is achievable only for DBSMA because it neither uses any omni-directional transmissions nor transmits directional messages in all the directions. Even with a single omni-directional transmission, the directional-back-off would not be possible. Also, if a directional antenna is used to transmit any packet in all directions (by sending it in different directions one by one as proposed in [10]) independent directional back-offs cannot be maintained. Thus the DBSMA architecture is inherently suitable for this enhancement that yields better performance as will be discussed later.

2) Neighbor Direction Detection: Till now we have assumed that the transmitter knows the direction that it has to use to communicate with the next hop destination. A good method to obtain this information is given in [10]. In this method, each node maintains one database entry for each 
neighbor. For each neighbor, the database entry at a node contains the direction that itself and a neighbor would use for communicating with each other. This information is gathered through by exchanging the direction information in control packets. This method can be adapted to DBSMA, however the details are left out for the sake of brevity.

\section{Benefits of DBSMA protocol}

DBSMA provides a natural solution to the hidden node problem by the use of a busy signal. In the description of DBSMA protocol, we have not assumed an ideal directional pattern. In DBSMA, the only information that a sender needs is the directions to reach different neighbors. Unlike other protocols that need exact location information and DNAV information to provide good spatial reuse, spatial reuse in DBSMA protocol is achieved through the use of the busy signal. If DNAV information is available, it could be used for more efficient operation. Specifically, using the DNAV information, a node can decide which directions it should skip when sweeping (for DNAV specified time). Potentially this can increase the channel utilization by decreasing the idle time between communications. In the design of most of the directional MAC protocols, a single directional pattern at all nodes is inherently assumed. Hence these protocols are not fully inter-operable among different types of antenna; however DBSMA protocol is. It is not necessary for all nodes to have the same type of antenna in the DBSMA protocol. Also the directional antennas need not be aligned with some reference direction.

\section{Simulation Results}

In this section, we compare the proposed DBack-off mechanism and the normal back-off mechanism. The network graph used for the simulations is given in Figure 4. The simulations are implemented in a time-slotted manner in which all the events are scheduled to start at the beginning of a timeslot. The timeslot width $(\sigma)$ is set to one count in the back-off window. The time required to listen/detect an invitation signal and change the listen direction for an idle node $(r)$ is also assumed to be equal to one timeslot. The transmission time for the RTS, CTS, and ACK messages is assumed to be equal to one timeslot. These values are chosen to approximately correspond to the DSSS (Direct Sequence Spread Spectrum) case in IEEE 802.11 with a rate at 11 Mbps. A data packet is assumed to last for 18 timeslots. We ignore SIFS and DIFS in the simulations.

The nodes are assumed to be greedy. This means that every node has always some data to send to all its neighboring nodes. Whenever a new transmission needs to be scheduled, a node selects a random neighbor from the set of free neighbors. The set of free neighbors is the subset of neighbors which can be reached via the directions that are not blocked by any other ongoing transmission. This information is obtained through the busy signal and the busy data/control channel information via listening in each direction. As two nodes can possibly communicate using multiple direction pairs as shown

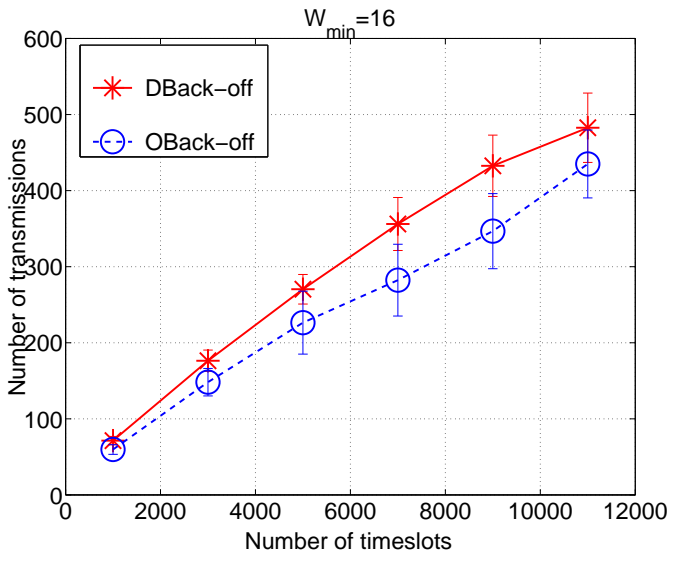

Fig. 5. Number of transmissions versus number of timeslots.

in Figure 4, the direction for communication is also chosen randomly from the set of unblocked directions through which the selected receiver node can be reached. In the DBack-off scenario, for every node the back-off window in each direction is maintained separately. In the second scenario, which is termed as OBack-off (Omni Back-off), for every node there is a single back-off window across all directions.

Figure 5 plots the number of successful transmissions versus the number of simulation timeslots. For each parameter setting, the simulations are performed 20 times. The plots show the mean values as well as the standards deviations (the total height of the vertical bars represents two standard deviations). From this figure we notice that there is a significant difference $(\approx 15-30 \%)$ between the number of succesful transmissions using the DBack-off mechanism and the OBackoff mechanism. We have observed a considerable difference between the average back-off values for different directions using the DBack-off mechanism and the back-off values using the OBack-off mechanism. This indicates the the DBackoff mechanism achieves higher throughput compared to the OBack-off mechanism.

Figure 6 plots the mean number of successful transmissions for different values of the minimum back-off window $(4,8,16,32,64)$. From the figure we notice that using a smaller minimum back-off window achieves considerably better performance. Quantifying spatial utilization corresponds to quantifying the number of simultaneous successful transmissions. If a particular direction is busy, a node equipped with directional antenna can try another direction to initiate a new communication. However, this can only be done after a new (directional) back-off timer expires. This observation along with the fact that the number of directional neighbors is smaller than the number of all possible neighbors, indicates that the minimum back-off window using directional antennas should be smaller compared to the back-off window used with omni-directional antennas.

We have modified the analysis presented in [12] to study the effect of overheads on the throughput in the directional MAC 


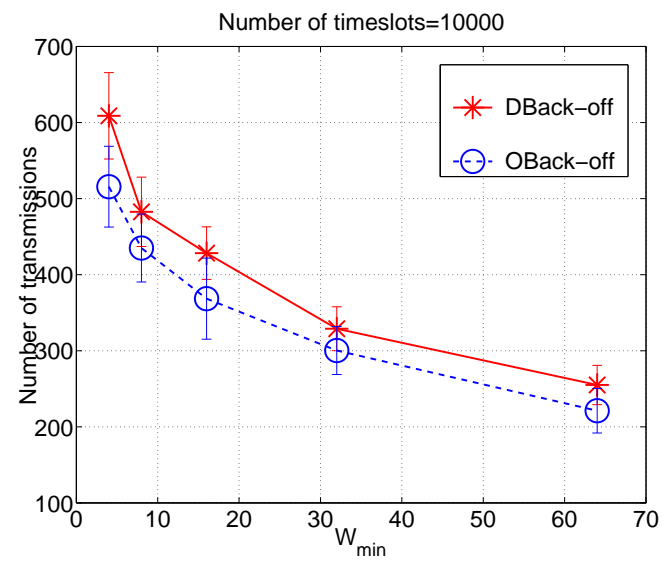

Fig. 6. Number of transmissions versus minimum back-off window.

protocols. The analysis methodology assumes that all nodes are neighbors. We consider two protocols namely DBSMA and DMAC (the protocol suggested in [10]). The throughput is represented by the fraction of the time the channel carries payload bits. Due to the space limitations we present only part of the numerical results in this paper, i.e., we show the effect of unequal rates for data and control packet transmission on throughput. We assume that the data rate is $11 \mathrm{Mbps}$ and the control packet rate is $1 \mathrm{Mbps}$. (As all nodes would not be neighbors in a normal scenario, the actual throughput would be higher due to spatial utilization.)

We first note that the throughput of the DBSMA protocol critically depends on the duration of the Invitation Signal. Assuming the duration of the Invitation signal is $10 \mu \mathrm{s}$, i.e., is equal to the duration of SIFS, Figure 7 plots the throughput for the two protocols versus the number of neighbors. From the graphs we notice that the throughput for the DMAC protocol is much smaller than the throughput of DBSMA. The main reason is that the DMAC protocol spends a lot of time sending RTS packets in all the directions. This points us to an important fact that if the average packet length is small, or if the control packet rate is smaller than the data packet rate then the DMAC protocol cannot achieve a good channel utilization.

\section{CONClusion}

In this paper, we considered the problem of designing a MAC protocol for directional antennas. We discussed various shortcomings of many MAC protocols suggested in the literature and motivated the need for a better one. We then proposed DBSMA, a simple yet efficient protocol based on the busy tone principle. DBSMA uses novel concepts of idle directional listening, beam sweeping, Invitation Signal and directional back-off windows. Using these features, DBSMA satisfies all our stated requirements for a good MAC protocol using directional antennas. DBSMA has a clear performance advantage over other protocols. As a part of the future work, we plan to analyze and simulate the performance of DBSMA

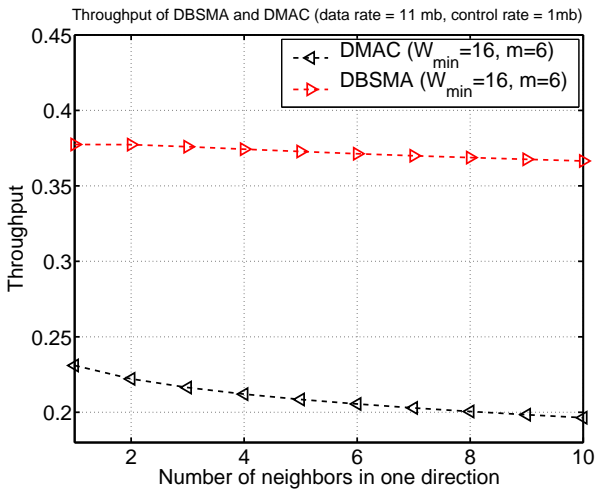

Fig. 7. Throughput of various MAC protocols: Using IEEE 802.11 DSSS parameters with $11 \mathrm{Mbps}$ data rate and $1 \mathrm{Mbps}$ control packet rate

protocols for various topologies and compare it with other protocols.

\section{ACKNOWLEDGMENT}

This work was supported in part by NSF grant (contract no. 0087266), and by Prof. Rosenberg's NSERC Discovery grant (RGPIN42023-37200).

\section{REFERENCES}

[1] F. A. Tobagi and L. Kleinrock, "Packet switching in radio channels: Part I,II," IEEE Transactions on Communications, vol. 23, pp. 1417-1433, December 1975

[2] H. Zhai, J. Wang, Y. Fang, and D. Wu, "DUCHA: A dual-channel MAC protocol for mobile ad hoc networks," in IEEE Workshop on Wireless Ad Hoc and Sensor Networks, November 2004.

[3] L. Wu and P. K. Varshney, "Performance analysis of CSMA and BTMA protocols in multihop networks (I), single channel case," in Proceedings of 11th International Conference on Computer Communications and Networks (ICCCN), 1999

[4] C. Wu and V. O. K. Li, "Receiver-initiated busy-tone multiple access in packet radio networks," in ACM SIGCOMM, 1987, pp. 336-342.

[5] S. Wu, Y. Tseng, and J.Sheu, "Intelligent medium access for mobile ad hoc networks with busy tones and power control," IEEE Journal on Selected Areas in Communications, vol. 18, pp. 1647-1657, Sept. 2000.

[6] R. Ramanathan, "On the performance of ad hoc networks with beamforming antennas," in ACM Mobihoc, Oct. 2001.

[7] R. R. Choudhary, X. Yang, N. H. Vaidya, and R. Ramanathan, "Using directional antennas for medium access control in ad hoc networks," in International Conference on Mobile Computing and Networking (MOBICOM), Atalanta, Georgia, USA, 2002.

[8] A. Nasipuri, R. E. Hiromoto, and S. Ye, "A MAC protocol for mobile ad-hoc networks using directional antennas," in Proceedings of the IEEE Wireless Communication and Networking Conference (WCNC), 2000.

[9] Y. B. Ko, V. Shankarkumar, and N. Vaidya, "Medium access control protocols using directional antennas in ad hoc networks," in IEEE Conference on Computer Communications (INFOCOM), March 2000.

[10] T. Korakis, G. Jakllari, and L. Tassiulas, "A MAC protocol for full exploitation of directional antennas in ad-hoc wireless networks," in Proceedings of ACM International Symposium on Mobile Ad Hoc Networking and Computing, Annapolis, USA, 2003.

[11] M. Takai, J. Martin, A. Ren, and R. Bagrodia, "Directional virtual carrier sensing for directional antennas in mobile ad hoc networks," in $A C M$ Mobihoc, June 2002.

[12] G. Bianchi, "Performance analysis of the IEEE 802.11 distributed coordination function," IEEE Journal on selected areas in communications, vol. 18, pp. 535-547, March 2000. 